\title{
Prólogo a \\ Prólogos para la memoria
}

Roberto Fernández Retamar ${ }^{\bullet}$

Casa de las Américas. Cuba

Dos aniversarios trascendentes coinciden en Cuba con los sesenta años de la Casa de las Américas: el del medio milenio de la ciudad de La Habana y el de los sesenta ańos de la victoria, el primero de enero de 1959, de nuestra Revolución, que menos de cuatro meses después creó la propia Casa de las Américas y puso a su frente a la legendaria heroína Haydee Santamaría, nuestro gran privilegio. Es por ello que cuando comenzamos a preguntarnos cuáles podrían ser las mejores formas de conmemorar en 2019 los sesenta años de nuestra institución, la compañera María Luisa (Chiki) Salsamendi, quien trabaja en el Archivo Memoria y en el lenguaje cubano corriente es lo que se considera una "histórica» dentro de la Casa de las Américas, tuvo la feliz idea de proponer que se reuniera en un volumen, dentro de la conmemoración, una selección tan amplia como posible de prólogos a libros publicados en la llamada originalmente nuestra Colección Literatura Latinoamericana, a la cual se añadió en 2004 el adjetivo Caribeña. En ella aparecen obras clásicas de nuestras letras. Enterada de la idea, otra "histórica» de la Casa, la compañera Silvia Gil, quien dirige el Archivo Memoria, se sumó con entusiasmo a la propuesta, y lo mismo hizo un compañero que nació años después de creada la Casa, Jorge Fornet, director del Centro de Investigaciones Literarias y codirector de la revista Casa de las Américas. Ellos, después de varias reuniones de trabajo, seleccionaron los textos que aparecen en este volumen, cuidando que no hubiera más de una obra de un autor, ni más de un mismo prologuista, lo cual, siendo justo, ha tenido como consecuencia que aquí no aparezcan prólogos a muchas obras de primer orden, de las cuales mencionaré tantas como sea posible al final de estas palabras. El trío casi musical me pidió que prologara el volumen, sin duda tomando en consideración que yo, que era un joven de veintiocho años cuando se creó la Casa de las Américas, donde leí la segunda conferencia que se ofreció en ella (fue sobre Andrés Bello), soy ahora, a mis ochenta y ocho, su trabajador más viejo. 
El compañero Jorge Fornet, aduciendo que no se puede ser juez y parte, opinó que no debía estar entre los seleccionados su prólogo a la novela Los días terrenales, tan discutida cuando apareció, del gran rebelde mexicano José Revueltas, quien fue encarcelado de nuevo en su país a raíz de los sucesos de 1968, hace ahora medio siglo. Pero convencí a Jorge de no prescindir de sus justas páginas, recordándole que, además del prólogo mío que aparece en este volumen (y, por supuesto, en el que intento hacer ahora), escribí para la Colección de marras otros, no recogidos aquí, sobre obras de cinco autores, y en tres casos escogí además las correspondientes antologías, aparte de asumir prólogos a obras fuera de la mencionada Colección, pero publicadas también por nosotros, de José Martí (Nuestra América), el argentino Ezequiel Martínez Estrada (Martí revolucionario), el mexicano y futuro Premio Cervantes José Emilio Pacheco (Fin de siglo y otros poemas) y el libro Materiales de la revista Casa de las Américas del sobre Julio Cortázar, cuyos textos seleccionó la cubana Xenia Reloba.

Cuando supe de la idea de Chiki, pensé de inmediato en un libro que creo poco visitado y he leído varias veces con deleite, como suelo leer las obras de su complicado e imprescindible autor, según saben quienes se han aventurado en mis papeles. Me refiero al libro del argentino Jorge Luis Borges Prólogos con un prólogo de prólogos, editado por Torres Agüero en Buenos Aires, en 1975. En las páginas iniciales de dicho libro, donde se reúnen prólogos escritos desde 1923 por Borges (quien sería Premio Cervantes), este aseguró: «Que yo sepa, nadie ha formulado hasta ahora una teoría del prólogo.» Esa sentencia se emitió en 1974, e ignoro si posteriormente ha sido formulada tal teoría, que en todo caso desconozco. Y después de nombrar y desechar varias definiciones, Borges ofreció la suya: «El prólogo, cuando son propicios los astros, no es una forma subalterna del brindis; es una especie lateral de la crítica», palabras citadas ya en el prólogo mío incluido en la presente selección.

Apenas es necesario decir que el volumen que intento prologar revela parte apreciable de la faena cumplida por la Casa de las Américas a lo largo de sus casi sesenta años. La Colección de la que provienen los textos aquí agavillados, junto con la de las obras merecedoras del Premio Literario que la institución ha venido otorgando desde 1960, es la más representativa suya en cuanto a la Literatura (en sus más diversas manifestaciones y enfoques), sin olvidar que la Casa aborda también, siempre en relación con nuestra América, otras manifestaciones culturales: Artes Plásticas, Música, Teatro. Cuenta, además, con un Centro de Estudios del Caribe, auspicia Programas de Estudios sobre la Mujer, Latinos en los Estados Unidos, Culturas Originarias de América, Afroamérica, otorga varios Premios, posee una nutrida Biblioteca, un Fondo Editorial (que antes fue llamado simplemente Editorial) con diversas Colecciones, cuatro Revistas, tres Galerías, una vasta Colección de obras de arte que lleva el nombre de la fundadora Haydee Santamaría, el Archivo Memoria, que atesora nuestro enorme epistolario, fotos y documentos relativos a la institución, el Archivo de la Palabra, con obras literarias grabadas por sus autores, del que se nutre la colección Palabra de esta América (sucesivamente de discos de vinilo, casetes y discos compactos que no mencionaré para no hacer excesivas estas páginas), y otro de piezas musicales, algunas de las cuales también darían lugar a discos, el portal informativo La Ventana, el programa de Televisión América en la Casa, una Red Comercial a lo largo de la Isla, y desde luego otros locales y dependencias. Por último, sin pretender agotar la amplia variedad de la Casa de las Américas, es necesario mencionar su proyecto juvenil Casa tomada, lleno de futuridad. 
La Casa de las Américas ha organizado incontables reuniones, de las que, por su crecido número, mencionaré solo los dos Encuentros de Intelectuales por la Soberanía de los Pueblos de Nuestra América, en los cuales nos honró con su presencia el compańero Fidel, como hizo en otras ocasiones, y además solía recibir cada año en el Palacio de la Revolución a los integrantes del jurado del Premio Literario Casa de las Américas. La revista Casa de las Américas, donde se habían publicado varios de sus textos fundamentales, a raíz de su desaparición le dedicó una entrega (Fidel siempre) que se agotó rápidamente. También estuvo en la Casa de las Américas el compañero Ernesto Che Guevara, quien en discurso pronunciado en Conferencia realizada en Punta del Este, Uruguay, en 1961, mencionó al Premio Literario Casa de las Américas (que entonces se llamaba Concurso Literario Hispanoamericano) como ejemplo de la «exaltación [que Cuba propicia] del patrimonio cultural de nuestra América Latina». Tras el asesinato del Che, la revista Casa de las Américas le consagró un número encabezado por una inolvidable carta de Haydee. Posteriormente publicamos una selección en dos tomos de Obras (1957-1967) del Che, que compilé y con las cuales se inició nuestra Colección Pensamiento de Nuestra América, los libros Evocación, de la cubana Aleida March, Ernesto Guevara, también conocido como el Che (editado conjuntamente con la Fundación Rosa Luxemburgo), del mexicano Paco Ignacio Taibo II y Materiales de la revista Casa de las Américas delsobre Ernesto Che Guevara, cuyos textos escogió Xenia Reloba y prologó el cubano Fernando Martínez Heredia, quien había obtenido el Premio Casa de las Américas con su libro Che, el socialismo y el comunismo. Una sala (la principal) del edificio central de nuestra Casa se llama Che Guevara.

Para saber cómo se inició nuestra Colección de obras clásicas de la Literatura Latinoamericana y Caribeña hay que remitirse a la compañera Marcia Leiseca, quien entró en la Casa de las Américas pocos meses después de hacerlo Haydee, de la cual se convertiría en su mano derecha: aunque para ser políticamente correcto debía decir su mano izquierda. Marcia, extremadamente joven, tenía ya una sorprendente sabiduría (que por supuesto iba a aumentar) y era de una belleza turbadora: como también lo eran Chiki, Silvia, la inolvidable María Rosa Almendros y otras muchachas de la institución. Ello hizo que el gran pintor chileno Matta, siempre ingenioso, hablase de «las casadas de las Américas», y que años después el escritor ecuatoriano Jorgenrique Adoum me preguntara seriamente si no me inquietaba trabajar junto a tantas beldades. Le expliqué que yo había encontrado en ellas las hermanas que no me dio la naturaleza, porque he tenido tres hermanos varones.

La que iba a ser la Colección que he venido mencionando, como otras realidades de la Casa de las Américas, fue idea de Marcia, quien la dio a conocer al argentino Ezequiel Martínez Estrada y sobre todo al guatemalteco Manuel Galich. Ellos fueron los primeros latinoamericanos no cubanos que vinieron a trabajar en la institución (uno en 1960 y otro en 1962), y obras de ambos habían recibido sendos Premios de la Casa de las Américas: Martínez Estrada en ensayo (en 1960) y Galich en teatro (en 1961). Don Ezequiel, como se le llamaba y cuyo magisterio he proclamado, escribió textos extraordinarios sobre Fidel y el Che recogidos en su libro En Cuba y al servicio de la Revolución Cubana, dejó inconclusa una obra monumental sobre José Martí y regresó en 1962, con salud muy quebrantada, a la Argentina, donde moriría dos años después. Al conmemorarse un año de su desaparición, le dedicó un número la revista Casa de las Américas. Además, la Colección de que hablo in- 
cluyó una antología póstuma de sus textos, y el Premio Extraordinario de Ensayo que anualmente otorga la Casa de las Américas lleva su nombre.

En cuanto a Galich, gran figura de la vida política y cultural de su país, le fue imposible volver a él, debido a los regímenes que Guatemala padeciera desde el derrocamiento por la CIA, en 1954, del gobierno progresista de Jacobo Arbenz, que a la sazón Galich representaba como diplomático en la Argentina, y permaneció en Cuba hasta su muerte en 1984. Fue subdirector de la Casa, fundador de la dirección de Teatro y de su revista Conjunto e integrante del comité de colaboración de la revista Casa de las Américas. Entre las obras suyas publicadas por la Casa se destacan la dedicada a los aborígenes americanos, Nuestros primeros padres, el ensayo Mapa hablado de la América Latina en el año del Moncada, y póstumamente un volumen de Páginas escogidas en la Colección que menciono. Además fue profesor en la Universidad de La Habana y colaboró en la prensa cubana. También lo aprecié mucho, mucho aprendí de él, tuve el triste privilegio de hablar ante su tumba recién cerrada, y en la ocasión le rindió homenaje la revista Casa de las Américas. Otra de las salas del edificio central de la Casa lleva su nombre.

Cuando razones ingratas en las que no vale la pena insistir llevaron a Marcia a abandonar la Casa de las Américas (por suerte temporalmente, pues regresaría años después y es su imprescindible vicepresidenta primera), en una de las reuniones del Consejo de Dirección en las que, antes de ser amordazado por presidirlo, yo tenía la mala costumbre de pasar versos más o menos risueños a las compañeras y los compañeros, hice llegar a Marcia esta décima, hecha con el auxilio involuntario de Federico García Lorca, y que por supuesto, como toda su familia nacida en el Consejo, no aparece en mis sumas poéticas:

\section{ÚLTIMOS VERSOS PARA TI EN ESTA MESA}

A Marcia Leiseca, cuando dejó la Casa de las Américas

Ya nada va ser igual,

Ni alegría ni tristeza:

Cuerpo seré sin cabeza,

Metal de voz sin metal,

Vaso que perdió el cristal,

Sombra que la luz rechaza,

Porque eres ave que pasa

(Que pasa no: que pasó),

Porque yo ya no soy yo,

Ni mi Casa es ya mi casa.

Volvamos a nuestros corderos, como dicen los franceses, o a mi canto llano, según el Quijote. Me refiero, claro está, a la Colección Literatura Latinoamericana y Caribeña. Las obras que la integran han sido propuestas, sobre todo, por quienes han trabajado en la Casa, cubanos y cubanas en su mayoría, pero también procedentes de otros países de nuestra América que no le han faltado nunca a la institución. (Hace años está con nosotros la compañera ecuatoriana María Elena Vinueza, directora de Música y una de nuestras vicepresidentas.) No es dable mencionar a todas y a todos, quienes a veces habían tenido que abandonar sus países de nacimiento por las feroces dictaduras implantadas en ellos siguiendo orientaciones del imperio. Y no solo es válido evocar a quienes prestaron su colaboración en 
nuestros locales. Haydee solía decir que no pocos trabajadores y trabajadoras de la Casa de las Américas se encontraban (y se encuentran) desperdigados por el subcontinente, e incluso por el planeta, lo que es una verdad indiscutible, y prueba de ello es la inmensa cohorte de escritores y artistas que han participado en los múltiples eventos y reuniones auspiciados por la Casa de las Américas, y cuyas numerosísimas obras nos enriquecen. Pero en representación de quienes trabajaron en nuestros locales voy a detenerme, como hice a propósito de Martínez Estrada y Galich, en otro caso: el del uruguayo Mario Benedetti.

La honda relación de Benedetti con la Casa, que mantuvo hasta el final de su vida, conoció dos momentos. Integró en 1966 el jurado del Premio Literario Casa de las Américas, formó parte del comité de colaboración de la revista Casa de las Américas y participó en el Encuentro con Rubén Darío, organizado por la Casa al conmemorarse en enero de 1967 el siglo de haber nacido el gran nicaragüense que inició la poesía moderna en lengua española. Tres de los compañeros que animaron aquel Encuentro (el excelente poeta mexicano Carlos Pellicer y los notables críticos Manuel Pedro González, hispano-cubano, y Ángel Rama, de Uruguay: este último muy vinculado con la institución e integrante del comité de colaboración de la revista Casa de las Américas) propusieron que se crearan en Cuba dos centros de estudios: uno relativo a la literatura de nuestra América y otro a la obra de José Martí. Invitado por Haydee, Benedetti aceptó dirigir, en el seno de la Casa de las Américas, el primero, con el nombre que propuso: Centro de Investigaciones Literarias, mientras el compañero Sidroc Ramos, director de la Biblioteca Nacional José Martí, creó, con el poeta y ensayista cubano Cintio Vitier a su frente, la Sala Martí, que años después daría lugar al Centro de Estudios Martianos, que me correspondió fundar, y del que Cintio sería hasta su muerte presidente de honor. Benedetti fue autor de cuantiosas y admirables obras, y su fecundísima labor al frente del Cil, como se lo conoce, hizo que no pocos de sus aportes conserven validez en nuestros días. Nostálgico de su país, regresó a él a principios de 1971, aunque lo seguimos considerando integrante de nuestro Consejo de Dirección. Sin embargo, ni él ni nosotros podíamos prever que Mario regresaría a la Casa cuando las sangrientas dictaduras militares proimperialistas implantadas, a raíz del atroz crimen chileno de 1973, en países de la América del Sur y el Plan Cóndor lo obligaron a abandonar sucesivamente, en peligro de ser asesinado, Uruguay, la Argentina de la Triple A y Perú, y volver, tras recibir una inmediata respuesta positiva de Haydee a una solicitud urgente suya, a la Cuba que consideraba con razón su segunda patria, y donde asumió de nuevo durante años sus responsabilidades en la Casa de las Américas. Durante su vida, el Cil (no dirigido ya por Mario) le dedicó una Valoración múltiple de su obra realizada por el cubano Ambrosio Fornet, integrante del comité de colaboración de la revista Casa de las Américas, y publicamos varias obras suyas. La revista Casa de las Américas le rindió homenaje a su muerte, y tiempo después se le dedicó el libro Materiales de la revista Casa de las Américas delsobre Mario Benedetti, escogidos por Xenia Reloba y prologados por la cubana Caridad Tamayo Fernández, directora de nuestro Fondo Editorial.

A propósito de la Colección Literatura Latinoamericana y Caribeña, me es materialmente imposible comentar el conjunto de sus títulos (en el momento en que escribo van a ser ciento ochenta y ocho), e incluso no es mucho lo que puedo decir sobre todos los prólogos incluidos en este volumen, por lo que solo mencionaré varios y desde luego sus correspondientes obras. En cuanto a estas últimas, aunque 
por razones obvias la gran mayoría procede de la América de lengua española, se ha querido que estén representadas también, en versiones castellanas, otras áreas de nuestra América: el Brasil (la primera obra de la Colección fue la novela $\mathrm{Me}$ morias póstumas de Blas Cubas, de Joaquim Maria Machado de Assis, traducida del portugués por Antonio Alatorre), el Caribe anglófono y el francófono.

Sobre los prólogos a las obras se han tomado en consideración, en un caso, afinidades electivas, dicho sea con licencia de Goethe. Así, la antología de cuentos de Horacio Quiroga fue escogida y prologada por Ezequiel Martínez Estrada, quien consagrara al uruguayo su magnífico libro El hermano Quiroga (Montevideo, 1957), donde confesó que Quiroga «me inició en la lectura de obras desagradables, que había considerado yo de menor cuantía, y extinguió en mí la lámpara mortecina de la poesía». Junto con el cubano Francisco Pividal, a Manuel Galich se debe, en considerable medida, la constante presencia en la Casa de las Américas del Libertador Simón Bolívar (cuyos Documentos seleccionó y prologó el entrañable guatemalteco), quien había dicho: «Para nosotros, la patria es la América», y también que «los Estados Unidos [...] parecen destinados por la Providencia para plagar la América de miserias a nombre de la libertad». El salvadoreño Roque Dalton, tan vinculado con la Casa, nos dio a conocer la dramática Visión de los vencidos, del sabio mexicano Miguel León Portilla, por lo que prologó nuestra edición de la obra. Roque recibió el Premio Casa de las Américas por su poemario Taberna y otros lugares, publicamos otros libros suyos, y póstumamente el Cil le dedicó la Valoración múltiple de su obra realizada por el uruguayo Horacio García Verzi, y apareció el libro Materiales de la revista Casa de las Américas de/sobre Roque Dalton, escogidos por los cubanos Sandra Valmaña y Aurelio Alonso, subdirector de la revista Casa de las Américas, y prologados por este. El haitiano René Depestre, admirable poeta él mismo, de quien Frantz Fanon citó versos en Los condenados de la Tierra y que formó parte del comité de colaboración de la revista Casa de las Américas, estaba particularmente capacitado para presentar al gran poeta de Martinica Aimé Césaire, el cual a su vez había prologado el libro del haitiano Végétations de Clarté. En otras de nuestras Colecciones publicamos el poemario de Depestre Un arcoiris para el Occidente cristiano (mención del Premio Casa de las Américas) y su ensayo Buenos dias y adiós a la negritud. En cuanto a Mario Benedetti, fue a nombre de la Casa de las Américas toda (Haydee vivía todavía, y su juicio fue trepidante) que prologó Cien años de soledad, ese Quijote del siglo xx editado por nosotros al año siguiente de su aparición inicial, en Buenos Aires, autorizados por su autor, el colombiano Gabriel García Márquez (a quien aún joven se le otorgaría el Premio Nobel), muchos de cuyos otros libros, con su anuencia, también publicamos, y el Cil le dedicó la Valoración múltiple de su obra realizada por el cubano Pedro Simón. Quizá su interés de entonces en la polémica llevó al joven colombiano Óscar Collazos, quien sustituyó a Benedetti al frente del Cil tras la primera partida de este, a prologar la peleadora e imaginativa novela del argentino Leopoldo Marechal Adán Buenosayres, que su compatriota Julio Cortázar fue de los muy pocos que la elogiaran al aparecer, y el cubano José Lezama Lima me comentó con aprecio muchos años antes de que viera la luz Paradiso. La chilena Rosario Carcuro seleccionó y prologó Cuentos, del dominicano Juan Bosch, a los que añadió unas páginas teóricas del autor sobre el cuento que habían interesado a García Márquez. En otra de nuestras Colecciones publicamos el magnífico libro de Juan Bosch De Cristóbal Colón a Fidel Castro. El Caribe, frontera imperial. El 
jamaicano Keith Ellis estudió y tradujo obras de Nicolás Guillén con sumo acierto, lo que lo autorizó a compilar y prologar Páginas escogidas del autor de El son entero y Prosa de prisa, a quien la revista Casa de las Américas dedicara una entrega al cumplir ochenta años, y el Cil una Valoración múltiple de su obra al cuidado de la cubana Nancy Morejón, exdirectora del Centro de Estudios del Caribe.

Algunos prologuistas latinoamericanos y caribeños han pertenecido a los mismos países de los autores cuyas obras presentaron, y en varias ocasiones propusieron incluir en la Colección. Un caso singular fue el del gran novelista peruano Mario Vargas Llosa, quien con justicia recibiría luego el Premio Nobel, y en la segunda mitad de los años sesenta del siglo pasado, en ejercicio de su colaboración, nos enviara dos textos sobre José María Arguedas, uno de los cuales lo publicó la revista Casa de las Américas y otro prologó la novela de este Los ríos profundos. Vargas Llosa, como es sabido, se apartó luego de la Casa de las Américas y de la izquierda en general, y pasó a ser defensor encarnizado de la derecha. Ya en esa posición, dio a conocer en 1978 su libro La utopía arcaica. José María Arguedas y las ficciones del indigenismo. De Arguedas, además de la novela mencionada, publicamos sus obras Katatay/Temblor y El sueño del pongo (que el cubano Santiago Álvarez llevó al cine con guión mío), el Cil le dedicó una Valoración múltiple de su obra al cuidado de su compatriota Juan Larco, y daría su nombre al Premio Extraordinario de Narrativa de la Casa de las Américas. Por su parte, el mexicano Emmanuel Carballo, quien formó parte del comité de colaboración de la revista Casa de las Américas, prologó la novela Al filo del agua, de Agustín Yáńez; el argentino David Viñas (cuya novela Los hombres de a caballo recibió el Premio Casa de las Américas, y que también integró el comité de colaboración de la revista Casa de las Américas) su Antología de Roberto Arlt; el chileno Enrique Lihn (merecedor del Premio Casa de las Américas por su libro Poesía de paso) las Poesías que escogió y prologó de Vicente Huidobro; el ecuatoriano Jorgenrique Adoum (el primer poeta en recibir el Premio Casa de las Américas, por su libro Dios trajo la sombra) los Cuentos que seleccionó y presentó de José de la Cuadra.

He dejado para el tercer lugar las menciones a prologuistas de Cuba, a quienes cito en el que creo que es el orden decreciente de sus edades. Dulce María Loynaz, la más joven de las poetisas hispanoamericanas que conmovieran nuestras letras en la primera mitad del siglo pasado, y en 1992 recibiera el Premio Cervantes, escogió y prologó con identificación Poesía, de la trágica uruguaya Delmira Agustini. A Dulce María el Cil le consagró una Valoración múltiple de su obra realizada por Pedro Simón. Nicolás Guillén, quien fue amigo personal del haitiano Jacques Roumain y tras su muerte le dedicara la memorable Elegía [...] en el cielo de Haití, prologó su novela Gobernadores del rocio, que tanto tiene que ver con nuestro país, y sobre la cual el cubano Tomás Gutiérrez Alea (Titón) realizó su filme Cumbite. José Lezama Lima correspondió al intenso acercamiento a su obra de Julio Cortázar prologando con su verbo regio la muy original novela de este Rayuela, que hubiera entusiasmado al Lawrence Sterne que escribió Tristram Shandy. A Cortázar, quien formó parte del comité de colaboración de la revista Casa de las Américas, además del libro póstumo con materiales de/sobre él que ya mencioné, la revista Casa de las Américas le dedicó un número a raíz de su muerte, así como dedicó otro en su centenario a Lezama, sobre cuya obra el Cil publicó una Valoración múltiple que apareció dos veces: una al cuidado de Pedro Simón y otra del cubano Roberto Méndez Martínez; además, el Premio Extraordinario de 
Poesía que otorga la Casa lleva su nombre. José Rodríguez Feo, vinculado a notables revistas culturales cubanas, encontró el rumbo definitivo de su vida profesional cuando asistió en la Universidad de Harvard, donde estudiaba, a conferencias sobre corrientes literarias hispanoamericanas ofrecidas por Pedro Henríquez Ureña, de quien se hizo cercano amigo y con quien mantuvo una interesantísima correspondencia en español e inglés parcialmente publicada en la revista Casa de las Américas. Con ese aval escogió y presentó ensayos del maestro dominicano. Un diálogo entre grandes poetas es el prólogo de Eliseo Diego (Premio Juan Rulfo) a la antología que realizara de la esencial chilena Gabriela Mistral (Premio Nobel). Y otro tanto debe decirse del prólogo de Cintio Vitier (también Premio Juan Rulfo) a la antología poética del fraterno nicaragüense Ernesto Cardenal (a quien el Cil dedicó una Semana de Autor). Graziella Pogolotti, quien formó parte del comité de colaboración de la revista Casa de las Américas y preside con acierto la Fundación Alejo Carpentier, prologó la gran novela del cubano El Siglo de las Luces, sobre la cual su compatriota Humberto Solás realizó un filme. También a Alejo Carpentier, quien recibió en 1977 el Premio Cervantes, consagró una entrega, al cumplirse un siglo de su nacimiento, la revista Casa de las Américas, y el Cil le dedicó una Valoración múltiple de su obra realizada por el cubano Salvador Arias. Lisandro Otero (Premio Casa de las Américas por su novela La situación, integrante del comité de colaboración de la revista Casa de las Américas y exdirector de nuestro Centro de Estudios del Caribe) prologó la novela Las lanzas coloradas, del venezolano Arturo Uslar Pietri. Leonardo Acosta prologó la impresionante novela del argentino Haroldo Conti Mascaró, el cazador americano, que el cubano Rapi Diego llevó al cine. Antonio Benítez Rojo (quien recibiera el Premio Casa de las Américas por su libro de cuentos Tute de Reyes y dirigiera nuestra Editorial) prologó, del raigal mexicano Juan Rulfo, los cuentos de El llano en llamas y la novela Pedro Páramo, ambos en un mismo volumen. A Luisa Campuzano, exdirectora del Cil, quien fundó y dirige en la Casa de las Américas el Programa de Estudios sobre la Mujer, correspondió seleccionar y prologar Obras escogidas en prosa y verso de la cubana Gertrudis Gómez de Avellaneda, a quien muchos y muchas consideramos la mayor escritora en idioma español del siglo xIx, y que la Real Academia Española perdió la ocasión de recibir en su seno como primera mujer de la entidad. Emilio Jorge Rodríguez (exdirector del Centro de Estudios del Caribe y Premio Casa de las Américas por su libro Una suave, tierna linea de montañas azules: Nicolás Guillén y Haiti) prologó la novela En el castillo de mi piel, del escritor de Barbados George Lamming, quien asesorara nuestro Centro de Estudios del Caribe y fue el primer autor en traer Caliban al Caribe, lo que hizo en su libro Los placeres del exilio, incluido más tarde en la Colección con natural prólogo mío. Fernando Martínez Heredia presentó una edición de Las venas abiertas de América Latina revisada por su autor, el uruguayo Eduardo Galeano (ganador dos veces del Premio Casa de las Américas con su novela La canción de nosotros y el testimonio Días y noches de amor y de guerra, así como del Premio Extraordinario de Narrativa José María Arguedas por Espejos. Una historia casi universal. Además publicamos otros libros suyos). Aún se recuerda que el presidente venezolano Hugo Chávez obsequió ante las cámaras un ejemplar de Las venas abiertas de América Latina al presidente estadunidense Barack Obama. Jesús Díaz, cuyo libro de cuentos Los años duros fue Premio Casa de las Américas, prologó los incisivos cuentos que Mario Benedetti llamó Montevideanos, como James Joyce había nombrado los suyos Dublineses. 
Trinidad Pérez Valdés, exdirectora del Cil y conocedora de la literatura del país en cuestión, donde fue diplomática, prologó la resonante novela Gran sertón: veredas, del brasileño João Guimarães Rosa. Guillermo Rodríguez Rivera, cuyo poemario El libro rojo fue recomendado en el Premio Casa de las Américas, seleccionó y prologó Poemas del más que secular chileno Nicanor Parra (Premio Cervantes). Víctor Casaus (mención del Premio Casa de las Américas por su testimonio Girón en la memoria) seleccionó y prologó Poesía del hermano argentino Juan Gelman (Premio Cervantes). Y el gran poeta Raúl Hernández Novás (a quien la Casa de las Américas entregó póstumamente el primer Premio Extraordinario de Poesía José Lezama Lima por su libro Amnios) seleccionó y prologó Poesía, del puertorriqueño Luis Palés Matos, parte de cuya obra, antes de recogerse en libro, se hizo sentir en la poesía negra cubana.

Entre las obras de la Colección Literatura Latinoamericana y Caribeña cuyos prólogos no se recogen en el presente volumen, y que en el primer párrafo anuncié que mencionaría al final de estas palabras, se encuentran libros de ensayos de los peruanos José Carlos Mariátegui y Antonio Cornejo Polar, los ecuatorianos Juan Montalvo y Benjamín Carrión, el colombiano Baldomero Sanín Cano, el mexicano Alfonso Reyes, el venezolano Mariano Picón Salas, el martiniqueño Frantz Fanon; poemarios del nicaragüense Rubén Darío, los chilenos Pablo Neruda (Premio Nobel), Pablo de Rokha, Gonzalo Rojas (Premio Cervantes), Violeta Parra, Enrique Lihn, los argentinos Baldomero Fernández Moreno, Alfonsina Storni, Raúl González Tuñón, Francisco Urondo, los colombianos León de Greiff y Luis Vidales, los mexicanos Amado Nervo y Carlos Pellicer, los puertorriqueños Juan Antonio Corretjer (también con una antología de prosa) y Julia de Burgos, el boliviano Ricardo Jaimes Freyre, el peruano César Vallejo, el ecuatoriano Jorge Carrera Andrade, el venezolano Vicente Gerbasi, el brasileño Carlos Drummond de Andrade, el uruguayo Mario Benedetti, el salvadoreńo Roque Dalton, Martín Fierro, antologías de poesía quechua, nicaragüense, gauchesca y Poesía trunca; libros de cuentos de los argentinos Roberto J. Payró y Julio Cortázar, los chilenos Baldomero Lillo, Mariano Latorre, Francisco Coloane, los venezolanos José Rafael Pocaterra, Julio Garmendia, Guillermo Meneses, el brasileño Joaquim Maria Machado de Assis, el ecuatoriano Pablo Palacios, el salvadoreño Salarrué, el colombiano Tomás Carrasquilla, el uruguayo Felisberto Hernández, Cuentos del Caribe, Quince relatos de la América Latina, Un siglo del relato latinoamericano; testimonios del mexicano Martín Luis Guzmán, los argentinos Lucio V. Mansilla, Domingo Faustino Sarmiento, Rodolfo Walsh, Ernesto Che Guevara (también cubano), el chileno Vicente Pérez Rosales; novelas de los brasileños Graciliano Ramos, José Lins do Rego, Mário de Andrade, Jorge Amado, Antonio Callado, Rubem Fonseca, los argentinos José Mármol, Ricardo Guiraldes, Ernesto Sabato (Premio Cervantes), Adolfo Bioy Casares (Premio Cervantes), Antonio di Benedetto, Ricardo Piglia (a quien el Cil dedicó una Valoración múltiple realizada por Jorge Fornet y una Semana de Autor), Juan José Saer, los uruguayos Enrique Amorim, Juan Carlos Onetti (Premio Cervantes), Mario Benedetti, los colombianos Jorge Isaacs y José Eustasio Rivera, los mexicanos Mariano Azuela y Carlos Fuentes (Premio Cervantes), los venezolanos Teresa de la Parra y Rómulo Gallegos, los ecuatorianos Jorge Icaza y Alfredo Pareja Diez-Canseco, los cubanos José Lezama Lima y Miguel Barnet, la peruana Clorinda Matto de Turner, el guatemalteco Miguel Ángel Asturias (Premio Nobel), el boliviano Augusto Céspedes, el chileno Manuel Rojas, el peruano Ciro 
Alegría, el paraguayo Augusto Roa Bastos (Premio Cervantes), el panameño Rogelio Sinán, la anglocaribeña Jean Rhys, el haitiano Jacques Stephen Alexis, el guyanés Jan Carew, la guadalupeña Maryse Condé (Premio Nobel alternativo), a quien el Cil dedicó una Semana de Autora; textos varios como Anales de los cakchiquekeles, Popol-Vuh. Libro del común de los quichés, libros de los peruanos Inca Garcilaso de la Vega y Ricardo Palma, los guatemaltecos Luis Cardoza y Aragón y Augusto Monterroso, el haitiano Jean Price Mars, el mexicano Juan José Arreola (a quien el Cil dedicó una Valoración múltiple realizada por la cubana Mirta Yáñez).

La Casa de las Américas, en vísperas de celebrar sus sesenta años de vida apasionada, se siente orgullosa de su existencia creadora (así haya asumido, como es habitual a lo largo de tantos años, actitudes ocasionales que ya no comparte), y en este caso particular de su Colección Literatura Latinoamericana y Caribeña, pero le interesan esencialmente las tareas que tiene por delante, y en gran medida serán obra no solo de jóvenes que hoy trabajan admirablemente en la Casa de Haydee y garantizan su pervivencia, sino también de los que José Martí, nuestro espíritu rector de siempre, llamó «compañeros desconocidos».

La Habana, 7 de diciembre de 2018 